\title{
Monetary costs of Alzheimer's disease in China: protocol for a cluster-randomised observational study
}

\author{
Fangyu $\mathrm{Li}^{1 \dagger}$, Shuoqi Chen ${ }^{1 \dagger}$, Cuibai Wei ${ }^{1^{*}}$ and Jianping Jia ${ }^{1,2,3,4^{*}}$
}

\begin{abstract}
Background: Alzheimer's disease (AD) is the most common type of dementia. International multilateral cost-of-illness (COI) studies have revealed that the cost of treating this disease is huge, which places a significant burden on patients' families and their healthcare systems. However, no such studies have been conducted in China. This study estimates the monetary costs of patients with AD in mainland China.

Methods: This study planned to start in October 2015 and to finish in March 2016. It covered 30 provincial, municipal, and autonomous regions in mainland China. The sites and research centres in each region were selected randomly. The participating sites include Tier 3 hospitals, psychiatric hospitals, geriatric hospitals, nursing homes, and residences. More than 2500 patients with AD and their caregivers from all of the 81 research centres will be enrolled to fulfil the calculated sample size. The monetary costs of $A D$, which include direct medical costs, direct non-medical costs, and indirect costs, are being collected using the electronic medical record system and residence health system at each site; face-to-face interviews are being performed when necessary. Descriptive statistics will be used to summarise the patient characteristics and generalised linear models will be developed to calculate the costs.

Results: The main findings will include national and per patient annual monetary costs of AD in China.

Conclusions: To the best of our knowledge, this is the first large-scale cluster-randomized observational study to estimate the economic burden of AD in Chinese patients. The methodology used was based on China's current healthcare system and is suitable for the purpose of the study. Because the burden of AD on patients, families, healthcare providers, and society is substantial and increasing, it is important and necessary to understand the economic burden caused by this disease.
\end{abstract}

Trial registration: Our trial was retrospectively registered on ClinicalTrials.gov, NCT02694445, registered on 02/26/2016

Keywords: Alzheimer's, Dementia, Cost of illness, Healthcare cost, Observational study

\section{Background}

The Chinese population is rapidly aging. The sixth national population census showed that there were 178 million people aged $\geq 60$ years in China, accounting for $13.26 \%$ of the total population [1]. The incidence of senile dementia (age of onset $\geq 65$ years) is $\sim 6 \%$, with Alzheimer's disease (AD) being the most common form, accounting for $\sim 65 \%$ of cases [2-5]. In 2014, an epidemiological study

\footnotetext{
*Correspondence: chuibainews@126.com; jjp@ccmu.edu.cn

Fangyu Li and Shuoqi Chen are co-first authors

${ }^{\dagger}$ Equal contributors

'Department of Neurology, Xuan Wu Hospital, Capital Medical University,

Beijing, People's Republic of China

Full list of author information is available at the end of the article
}

that encompassed 30 cities/towns and 45 rural areas from 5 representative Chinese provinces demonstrated that the incidence of dementia and $\mathrm{AD}$ in people aged $\geq 65$ was 5.14 and $3.21 \%$, respectively. Furthermore, the prevalence was significantly higher in rural areas compared to urban populations [6].

The monetary costs of AD have been studied worldwide. International multilateral cost-of-illness (COI) studies can identify and measure all of the costs associated with any specific disease including the direct, indirect, and intangible costs; [7] such studies were designed to provide an estimate of the economic burden of disease [7]. A recent study indicated that the cost of $\mathrm{AD}$ is substantial and 
increases with disease severity [8]. Between 2009 and 2010, a cross-sectional survey conducted in the United Kingdom, which included 249 AD patients from 18 cities, revealed that the total monetary costs of AD were $\sim £ 4000$ per citizen every 3 months [9]. Moreover, the annual costs associated with AD were $\$ 100$ billion in the United States, of which $\$ 24.6$ billion was for healthcare (including caregiver burden and the cost of medical care) [10]. In 2014, a French study showed that the average total monthly costs of AD were $€ 2450$ using the proxy good method, and $€ 3102$ using the opportunity cost method [11]. In the same year, a study in Finland indicated that the medical costs of an AD patient were $€ 23,059$ per person per year [12]. Thus, AD creates a heavy burden to both patients' families and society and is also a huge challenge to economic development and elderly healthcare. However, to date, studies on the monetary costs of AD have mostly been performed in developed countries such as the United States and United Kingdom, and there have been no large-scale national surveys on the economic burden caused by this disease in developing countries such as China. Because the burden of $\mathrm{AD}$ on patients, families, healthcare providers, and society is substantial and increasing $[13,14]$, our large-scale national study in China is important and necessary.

\section{Methods \\ Study design}

This study is a multicentre study with a large sample size that began in October 2015. Before beginning the study, the protocol and case report form (CRF) were designed using an operator manual, and advice was sought from international scholars. The study design and procedures are illustrated in Fig. 1.

\section{Distribution of investigation sites and inclusion criteria}

This study covered 30 provincial, municipal, and autonomous regions in mainland China, with the exception of Tibet, Hong Kong, and Macao. The target sites included Tier 3 hospitals, mental health centres/psychiatric hospitals, gerontology hospitals, nursing homes/gerocomiums/care facilities, and both urban and rural residences. The study sites in each region were selected randomly. However, each site must meet the following pre-specified criteria: (1) Tier 3 general hospitals, mental health centres, psychiatric hospitals or gerontology hospitals with memory clinics,

Stage 1: Preparation

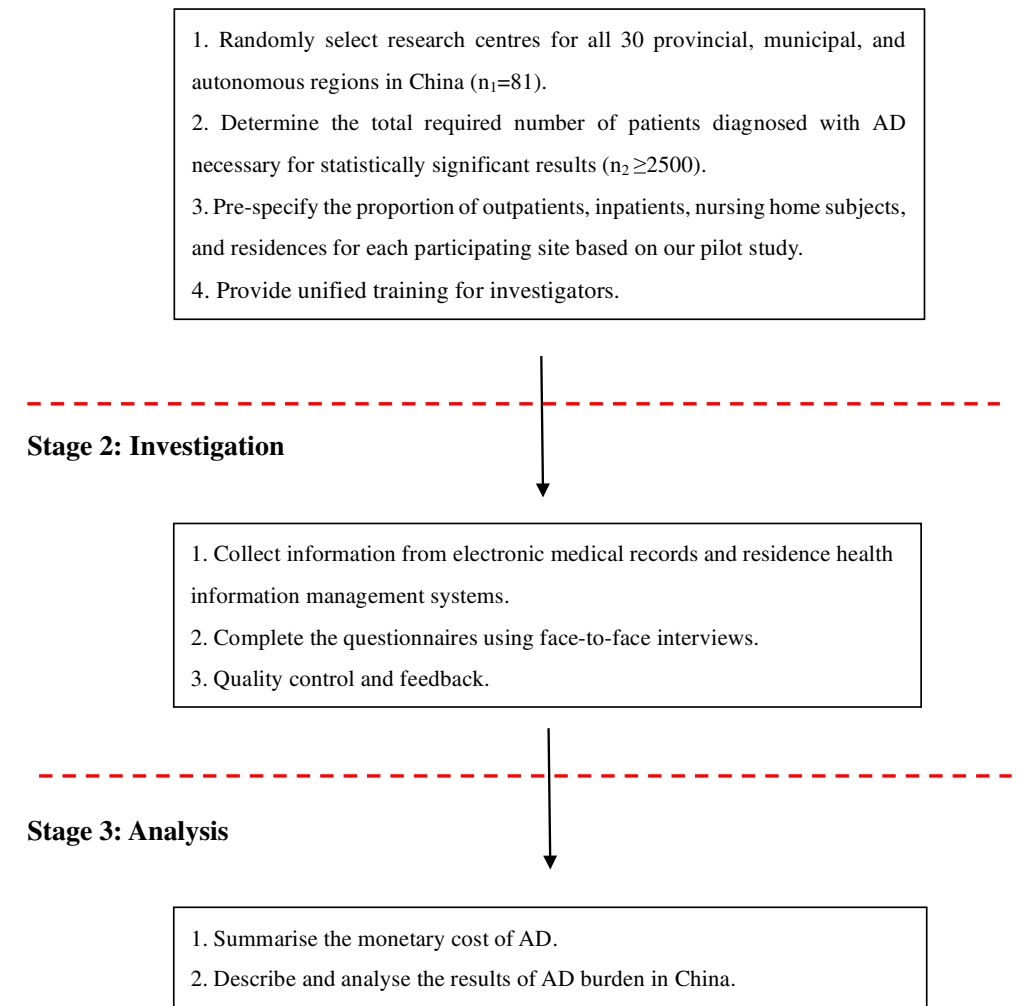

Fig. 1 Flow chart of the monetary costs of Alzheimer's disease in China. Note: $n_{1}$, the number of research centres; $n_{2}$, the number of patients with $A D$ 
dementia specialists, psychological evaluation divisions, AD-relevant diagnostic equipment (e.g., computed tomography $[\mathrm{CT}], 1.5 \mathrm{~T}$ or above magnetic resonance imaging [MRI]), and laboratories with the ability to examine samples that may aid in the diagnosis of AD. (2) Nursing homes/ gerocomium/care facilities with dementia specialists, $>100$ beds, and the ability to admit patients with AD. (3) Residences with $>500$ residents, a residence health system (e.g., electronic health records), active resident or village committees, and staff who can assist with the study. (4) Patients who fully understand the protocol and agree to participate in the study.

The study included 81 research centres that meet the inclusion criteria (Fig. 2).

\section{Criteria for AD patients}

Because we investigate the direct and indirect monetary costs of $\mathrm{AD}$, strict inclusion and exclusion criteria for $\mathrm{AD}$ are essential. The following patients are being included: those who are newly diagnosed with $\mathrm{AD}$ in this study or who have been previously diagnosed, patients aged $\geq$ 60 years with normal or corrected vision and hearing, and those who are able to provide an informed consent form signed by himself/herself or a legal guardian. Dementia is diagnosed according to the criteria described by the Diagnostic and Statistical Manual of Mental Disorders, Fourth Edition, Text Revision (DSM-IV-TR) [15]. The diagnosis of $\mathrm{AD}$ is made using the National Institute of Neurologic and Communicative Disorders and Stroke and the Alzheimer's Disease and Related Disorders Association (NINCDS-ADRDA) [16]. The diagnosis also required to meet the following: a Modified Hachinski Ischemic Scale (MHIS) score $\leq 4$ [17], an Activity of Daily Living Scale (ADL) score $\geq 23$ [18], and a Geriatric Depression Scale (GDS) score < 11 [19]. Neuroimaging MRI is used to support the $\mathrm{AD}$ diagnosis (atrophy in the medial temporal lobe and a white matter lesion score $\leq 2$ according to the Fazekas criteria) [20,21]. The severity of $\mathrm{AD}$ is estimated according to the mini-mental state examination (MMSE) scale [22] (for illiterate dementia mild, 21-24 points; moderate, 11-20; severe, $\leq 10$. For literate dementia: mild, 16-19 points; moderate, $8-15$; severe, $\leq 7)$. The diagnosis of $\mathrm{AD}$ is confirmed by brain $\mathrm{CT}$ or MRI scans and laboratory tests to rule out any significant

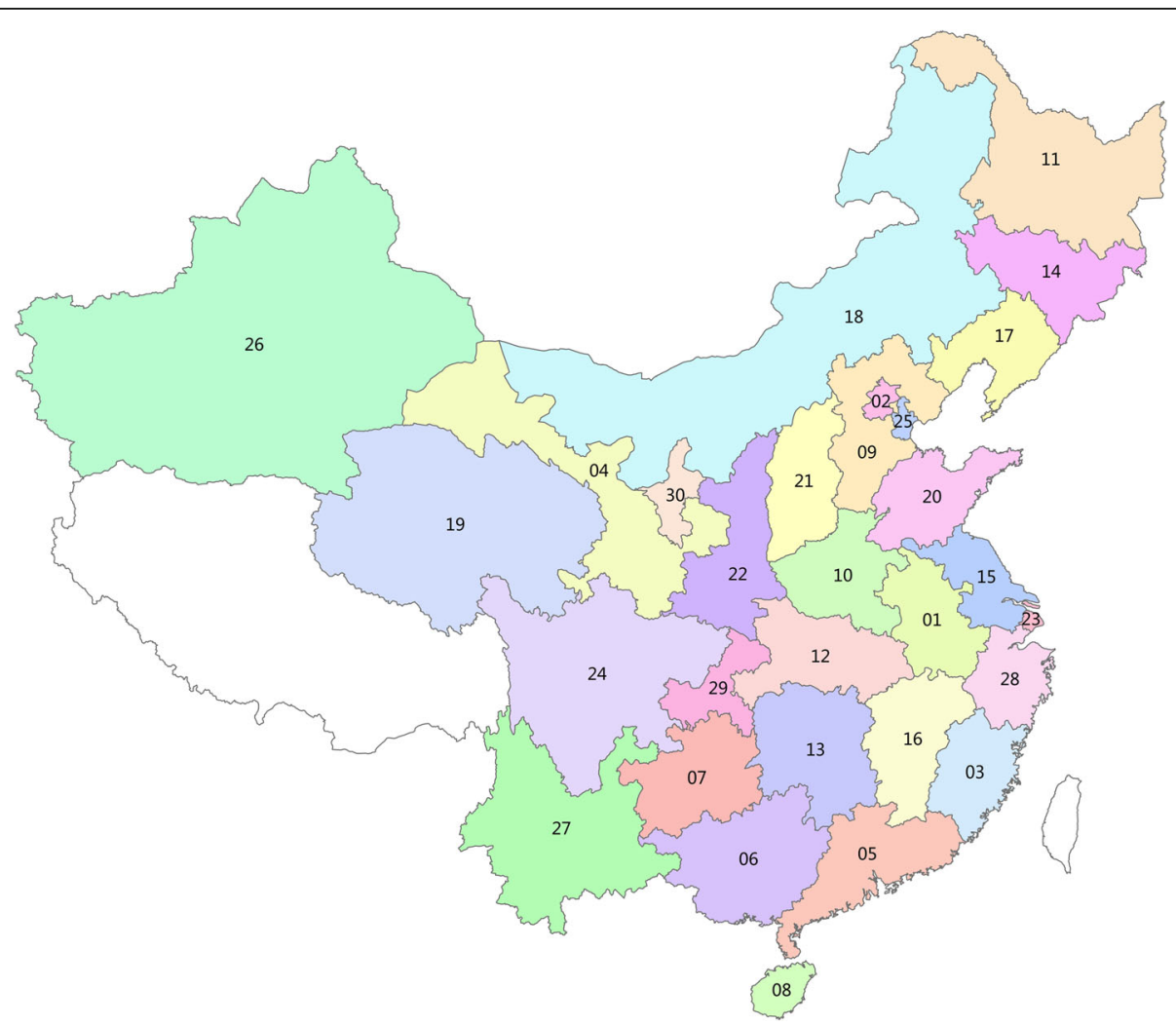

Fig. 2 The distribution of investigational centres in China. Notes: 01, Anhui Province; 02, Beijing City; 03, Fujian Province; 04, Gansu Province; 05, Guangdong Province; 06, Guangxi Autonomous; 07, Guizhou Province; 08, Hainan Province; 09, Hebei Province; 10, Henan Province; 11, Heilongjiang Province; 12, Hubei Province; 13, Hunan Province; 14, Jilin Province; 15, Jiangsu Province; 16, Jiangxi Province; 17, Liaoning Province; 18, Inner Mongolia Autonomous; 19, Qinghai Province; 20 Shandong Province; 21, Shanxi Province; 22, Shaanxi Province; 23, Shanghai City; 24, Sichuan Province; 25, Tianjin City; 26, Xinjiang Autonomous; 27, Yunnan Province; 28, Zhejiang Province; 29, Chongqing City; 30, Ningxia Autonomous 
comorbidities. Patients will be excluded if they meet any of the following criteria: presence of vascular dementia or dementia caused by other factors such as major depression or other major psychiatric illnesses, thyroid dysfunction, encephalitis, multiple sclerosis and other dementias such as frontotemporal dementia (FTD) [23], dementia with Lewy bodies (DLB) [24], and Parkinson's dementia (PD); [25] when MRI and laboratory tests (e.g., blood cell counts, liver and renal function tests) did not support or rule out a diagnosis of AD; and a history of alcoholism or drug abuse.

\section{Sample size}

The sample size was calculated using the equation, $n$ $=\frac{U_{1-\alpha / 2}^{2} S^{2}}{\delta^{2}}$ where $S$ was the stand division and $\delta$ was the allowable error. The planned sample size was generated based on previous data obtained from an economic evaluation of an AD-type dementia study [26]. Based on the standard deviation (SD) of AD-related costs per patient per year $(11,037)$ and an allowable error of 500 , the total sample size required was 1,872 . Therefore, the planned total sample size in this study was $\sim 2,500$ to allow for a defective rate of $25 \%$. Before starting the study, information was surveyed from all randomly selected participating sites, including the number of dementia physicians at each site, the number of AD patients, and the proportion of outpatients and inpatients in the last year. Based on these data $20-40$ patients with AD will be assigned for each site. The sites include hospitals (Tier 3 hospitals, gerontology hospitals, mental health centres and psychiatric hospitals), nursing homes, gerocomiums, care facilities, and residences.

\section{Cost classification}

The AD-associated costs are defined as direct medical costs, direct non-medical costs, and indirect costs (Table 1).

\section{Direct medical costs}

The direct medical costs include outpatient costs, hospitalisation costs, and out-of-pocket expenses for healthcare medications. Outpatient costs include the reason for the visit (due to $\mathrm{AD}, \mathrm{AD}$-related symptoms, or comorbidities), the number of outpatient visits in the past 3 months, and the cost of the last clinic visit in the same 3-months period. The cost of clinic visits includes registration (general physicians or specialists), diagnostic procedures (e.g., consultation, scale evaluation), imaging (e.g., CT, MRI, SPECT, and PET), laboratory tests, medication, and non-drug treatment (such as rehabilitation treatment). The outpatient medication costs are divided into three categories: anti-dementia drugs, medications for the treatment of AD-related symptoms
Table 1 Classification of AD-associated costs

\begin{tabular}{ll}
\hline AD-associated costs & Subgroup costs \\
\hline Direct medical cost & outpatient \\
& hospitalisation \\
& out-of-pocket \\
& transportation, \\
Direct non-medical cost & accommodation, \\
& and meals \\
& nourishment \\
& healthcare equipment \\
Indirect cost & formal care \\
& informal care \\
& intangible cost \\
\hline
\end{tabular}

(e.g., sleep disorders, psychiatric symptoms, and gastrointestinal symptoms caused by medications), and the treatment of comorbidities. For anti-dementia drugs the specific medications used and their corresponding costs are also investigated. For comorbidity-associated costs, the types of comorbidity and the associated costs are recorded. Investigations into the cost of hospitalisation included the reason for hospitalisation (AD, AD-related symptoms, or comorbidities), how many times a patient was hospitalised in the past 12 months, and expenditures on the last hospitalisation within the past 12 months. The hospitalisation expenditure includes the cost of the ward bed, formal care, examinations, laboratory tests, treatment (such as rehabilitation, massage, and others), and medications. The term "self-bought medications" means that the patient or their caregivers purchased the medications outside the hospital (out-of-pocket spending). Questions include how many times medications were purchased in the past month and the total expenditure during the same period. The above data also include information regarding the patients' payment method (including medical insurance for urban workers or town residents, rural cooperative medical insurance, commercial insurance and self-funded) and any out-of-pocket expenses.

\section{Direct non-medical costs}

The direct non-medical costs include the cost of transportation, accommodation, and meals when visiting a physician, the cost of nourishment and healthcare equipment in the patient's daily life, and formal care fees. The cost of daily nourishment, such as calcium, vitamin, protein powder, and fish oil supplements, include the number of times items were purchased and the amount spent in the last 12 months. The cost of healthcare equipment refers to supplies, such as positioning watches, bracelets, and wheelchairs, used in the last 12 months. Formal care costs are defined as the cost of nursing care given by professional 
caregivers for patients in nursing homes/gerocomiums/care facilities or at home, including the number of hours of care and the average monthly expenses [27].

\section{Indirect costs}

Indirect costs include monetary loss caused by the patients' inability to work, reduction of informal caregivers' income, treatment of the mental suffering of caregivers, and treatment of unexpected injuries in AD patients or their caregivers. Because the AD patients in this study are aged $\geq 60$ years, most of whom are retired as per China's national regulations, their major source of income is retirement pensions. Therefore, the decrease in a patients' income is not considered, because it is independent of patients' health status. Informal caregivers are mostly the relatives or unpaid nonrelatives of patients with no agency affiliation [28]. Investigation of the informal caregivers include the length of time (hours) provided by informal caregivers; the relationship between the caregivers and AD patients; sex, age, and monthly job losses for employed subjects (hours); and monthly salary. Cost for the treatment of mental suffering of caregivers refers to any symptoms triggered or worsened by taking care of $\mathrm{AD}$ patients over the past 12 months. The monetary loss caused by injuries to $\mathrm{AD}$ patients includes outpatient, examination, medication, transportation, and accommodation costs.

\section{Others}

To understand the relationship between $\mathrm{AD}$ costs and other factors, parameters such as social demographics and disease details are also investigated. The social demographics include sex, age, education level, marital status, occupation, salary, and the number of children. The disease details include age at onset of AD, clinical evaluations (based on ADL, GDS, MMSE and neuropsychiatric inventory [NPI] [29]), and comorbidities (e.g., stroke, diabetes, heart disease, hypertension, hyperlipidemia, lung diseases, cancer, arthritis, and other diseases of the nervous system).

\section{Data collection procedure}

Electronic medical record systems are being used to identify elderly patients diagnosed with $\mathrm{AD}$ among outpatients (in the last 3 months), inpatients (in the last 12 months), those living in urban residences and rural residences with their families, and residents of nursing homes, gerocomiums, or care facilities. The diagnosis of AD is re-confirmed by dementia specialists before the start of the study. The intensive dementia training program conducted in national and regional centres included both theory and practice. It comprised the study methods, assessment of the scales and internationally accepted diagnostic criteria of dementia and its subtypes, neuroimaging (CT and MRI), and standard procedures for diagnosis. Investigators are contacting patients and re-confirm the accuracy of the AD diagnosis according to the inclusion and exclusion criteria. Neuroimaging and laboratory tests will be performed when necessary. New patients are also being enrolled during the study period. Face-to-face questionnaires will be administered to $\mathrm{AD}$ patients and their caregivers when the data and information could not be collected through electronic medical record or residence health systems. The surveys are being administered by investigators to ensure the accuracy of the diagnosis and data.

\section{Measures \\ Total cost per patient per year}

The total cost per patient per year is determined by adding the annual direct medical costs, direct nonmedical costs, and indirect costs for each patient. When calculating the cost of the recuperation fee for patients who required nursing care in nursing homes, gerocomiums, or care facilities, $8 \%$ of the basic cost is subtracted to reflect the AD patient's basic cost of living [30]. Two approaches are being used to estimate the monetary loss of informal caregivers. For the informal caregivers who have no stable job before taking care of the $\mathrm{AD}$ patients, the average labour market income reported by each research centre is being multiplied by the amount of time spent being a caregiver to obtain the monetary loss. For individuals with a stable job before taking care of the AD patient, the monetary loss is being obtained by multiplying the hourly salary before they stop working by the time spent (hours) being a caregiver. All of the costs will be converted into the average annual cost.

\section{Current total number of patients with $A D$ in China}

A model is established to estimate the total number of Chinese patients with AD in 2015 based on the literature. The total number of patients with $\mathrm{AD}$ is defined as $\mathrm{M}$ and the percentage of $\mathrm{AD}$ patients who received antidementia treatment is defined as $\mathrm{d} \%$. Therefore, the number of anti-dementia treated patients $\left(M_{1}\right)$ is $M \times$ $\mathrm{d} \%$, and the number of patients not treated for dementia $\left(M_{2}\right)$ is $M \times(1-d) \%$. Based on previous reports, the percentage of $\mathrm{AD}$ patients who are treated for dementia is $10-30 \%$ [31, 32]. Epidemiological studies have revealed that the number of $A D$ patients in China aged $\geq 60$ years in 1990,2000 , and 2010 was 1.93 million, 3.71 million, and 5.69 million, respectively $[8,33,34]$. A linear regression model is used to estimate the age-specific prevalence of $\mathrm{AD}$ in 2015 by recording the prevalence of $\mathrm{AD}$ among different-aged patients. By multiplying the percentage of $\mathrm{AD}$ among elderly individuals aged $\geq$ 60 years by the total national elderly population (aged $\geq$ 
60 years) in 2015, we obtain the estimated total number of $\mathrm{AD}$ patients of China, which is defined as $\mathrm{M}$.

\section{Per capita annual cost of AD patients (who accepted or did not accept treatment)}

The cost associated with treating dementia patients $\left(\mathrm{F}_{1}\right)$ is divided into two groups: "outpatient costs" and "hospitalised or inpatient costs." $F_{1 \_1}$ is defined as the average annual cost in the inpatient group (average annual cost $\times$ the number of inpatients annually), and $\mathrm{F}_{1 \_2}$ is the average annual cost in the outpatient group (average annual cost $\times$ the number of outpatients annually). Patients described as not accepting treatment are those who did not select either "outpatient service costs" or "hospitalised costs" in their questionnaire. $\mathrm{F}_{2}$ is defined as the average annual cost per $\mathrm{AD}$ patient who did not accept treatment.

\section{Nationwide cost due to $A D$}

A pilot study is performed, which reveal that the average annual ratio of inpatients to outpatients in different hospitals is $\sim 1: 6$. Hence, the number of patients who undergo treatment nationwide $\left(\mathrm{M}_{1}\right)$ is multiplied by $1 / 7$ or $6 / 7$ to obtain the number of hospitalised patients with $\mathrm{AD}\left(\mathrm{M}_{1 \_1}\right)$ and the number of outpatients with $\mathrm{AD}$ $\left(\mathrm{M}_{1 \_2}\right)$, respectively. Thus, the total national annual cost of patients who accept treatment is $M_{1 \_1} \times F_{1 \_1}+M_{1 \_2} \times$ $\mathrm{F}_{1 \_2} \cdot \mathrm{M}_{2}$ is defined as the number of patients who did not accept treatment, and this value is multiplied by $\mathrm{F}_{2}$ to obtain the total annual national cost of patients who do not accept treatment. Therefore, the total annual national cost of patients with $\mathrm{AD}$ is $\mathrm{M}_{1 \_1} \mathrm{x} \mathrm{F}_{1 \_1}+\mathrm{M}_{1 \_2} \mathrm{x}$ $\mathrm{F}_{1 \_2}+\mathrm{M}_{2} \times \mathrm{F}_{2}$. To estimate the costs attributable to AD, generalised linear models (GLMs) related to coexisting conditions (stroke, diabetes, heart disease, hypertension, lung disease, cancer, arthritis, and psychiatric problems) and demographics (sex, age, educational level, marital status, and household income) are used. Moreover, we estimate the total numbers of people who will be living with AD in 2020, 2030, 2040, and 2050 to predict the total annual cost of $\mathrm{AD}$ in future decades. Few largescale national surveys of the monetary cost or economic burden of $\mathrm{AD}$ have been conducted in China, or indeed in Asia. To remedy this lack of data, we also systematically review $\mathrm{COI}$ studies of $\mathrm{AD}$ that report annual costs per patient. This will enable estimation of the proportion of the global burden of AD costs faced by China. Furthermore, it will enable comparison of the economic burden of $\mathrm{AD}$ in developed and developing countries.

\section{Statistical analysis}

Descriptive statistics

Descriptive statistics (means \pm SDs or frequencies) will be used to summarise the patient characteristics, including age, sex, years of formal education, time since AD diagnosis, marital status, living location (urban or rural), number of children, baseline MMSE score, baseline ADL score, number of comorbidities, number of caregivers, and information about the caregivers (such as sex, age, relationship to patient, and employment status). Mean values and ranges will be also presented because cost data are commonly positively skewed and the median cost could be zero for highly skewed data.

\section{Calculation of costs due to $A D$}

Different distributions will be considered for the monetary costs associated with $\mathrm{AD}$, including normal, lognormal, exponential, and gamma. To compare the costs of different subgroups, $t$-tests or Kruskal-Wallis analysis of variance will be used. For base case analyses, GLMs will be developed using the gamma distribution with a log-link function [35]. Models will be developed with the total cost per person per year as the dependent variable, and independent variables will be selected using a backward selection method $(P>$ 0.05 for removal), with patient age, patient sex, and MMSE severity all forced to remain in the model. Considering the diverse of research centres sites, we also added the sites as a covariant in the analysis. The variables used for backward selection will be years of formal education, time since AD diagnosis, marital status, location of residence (urban or rural), number of children, baseline MMSE score, baseline ADL score, the number of caregivers, the number of comorbidities, and caregiver demographics.

\section{Sensitivity analyses}

Sensitivity analyses will be conducted to examine the robustness of the results from the base case model and explore correlations between certain factors that could impact each other. First, the total costs of dementia will be estimated using the prevalence rates derived from a systematic review. Then the minimum and maximum salaries (rather than the average) will be used to estimate the relative costs. The total costs will be estimated by assuming that 70 and $99 \%$ of patients with $\mathrm{AD}$ are living at home, instead of $86 \%$ in the primary option. Furthermore, different costing caregiver time will be used to estimate the total cost. All of the analyses will be performed using $\mathrm{R}$ software version 3.2.3.

\section{Quality control}

The questionnaire and standard operating standards procedures were formulated based on previous studies and by consulting relevant experts internationally and domestically. All of the staff working on this study were trained to ensure that standard operating procedures were followed, and all of them were required to pass an examination 
before beginning data collection. A pilot study was conducted in five randomly selected hospitals and residences to evaluate the feasibility and reliability of the questionnaire and to identify any potential problems.

\section{Results}

This study began in October 2015 and will finish in March 2016. The main findings will be the per patient and national annual monetary costs of AD in China. The costs include direct medical, direct non-medical, and indirect costs. Data of the subgroup costs will also be reported. Social demographics, illness and treatment of $\mathrm{AD}$, scale scores, and comorbidities will be shown in a table at last.

\section{Discussion}

To the best of our knowledge, this is the first study on $\mathrm{AD}$ cost that encompassed nearly all of the provincial, municipal, and autonomous regions in mainland China. To complete this survey, it is crucial to prepare a thoughtful and well-designed protocol. In the study design phase, we referred to an international popular survey method and adjusted it to China's national conditions. We also tested the protocol in a pilot study; the results suggested that this protocol can be used in China.

This protocol includes measures specific to problems that exist in China. First, China has the largest population of any country, and the varying economic development among regions [36] might result in introduction of bias. To avoid this, the study includes 30 of the 33 provincial, municipal, and autonomous regions in mainland China. This ensures that both poor and rich areas are covered to balance the gap between them because people in poor areas may have more health problems [37]. Second, in China, hospitals with physicians for patients with AD include Tier 3 general hospitals, gerontology hospitals, mental health centres, psychiatric hospitals, and other institutions such as nursing homes, gerocomiums, and care facilities [36]. Therefore, all types of institutions are included in the current investigation to ensure representation of the diversity of AD costs among hospitals and institutions. The hospitals and institutions are selected randomly to reduce the test error. Third, the target population of this survey is patients with AD. Accurate diagnosis of $\mathrm{AD}$ is required for calculation of the true monetary cost of the condition. AD patients diagnosed according to the NINCDS-ADRDA criteria are enrolled in the study. In particular, to ensure accuracy, the diagnosis of patients diagnosed with $\mathrm{AD}$ prior to the start of this study was reconfirmed. In addition, the project team comprises a group of dementia physicians, who are trained in the diagnostic processes for dementia and $\mathrm{AD}$ to guarantee the quality and consistency of the diagnosis. Fourth, for accurate determination of the costs associated with $\mathrm{AD}$ patients, electronic records systems are used to determine the costs of all treatment items. This measure guarantees the absence of error. Therefore, the current protocol facilitates determination of the cost burden of $\mathrm{AD}$ patients in China.

We carefully consider cost classifications for the total cost of $\mathrm{AD}$. Cost in the present study includes direct medical costs, direct non-medical costs, and indirect costs. The cost classification used was in accordance with those that are commonly used internationally [11, 38]. This strategy is intended not only to ensure that the results cover all aspects of costs for $\mathrm{AD}$ patients but also to enable comparison with findings from developed countries. In addition, the cost of $\mathrm{AD}$ includes both $\mathrm{AD}$ patients and their caregivers. Our previous study revealed that $84.9 \%$ of $\mathrm{AD}$ patients are cared for by non-professional caregivers at home [39]. This is because China faces a shortage of nursing homes, and the Chinese tradition of filial piety mandates that when they lose the ability to live independently, patients should be cared for by their families. Therefore, it is important to include caregivers in calculating $\mathrm{AD}$ costs. These procedures are conducted to ensure the accuracy, reliability, and credibility of data on AD in China.

\section{Conclusion}

In conclusion, this study provides an important basis for calculation of the annual cost of $\mathrm{AD}$, will assist the understanding of the government and policy makers of the position and role of healthcare in the national economy and social development, and will enable formulation of a national plan for dementia. In addition, the results will facilitate evaluation of the effectiveness of the national funds allocated to AD research.

\section{Abbreviations}

AD: Alzheimer's disease; ADL: Activity of daily living scale; COI: Cost of illness; CRF: Case report form; CT: Computed tomography; DLB: Dementia with Lewy bodies; DSM-IV-TR: Diagnostic and Statistical Manual of Mental Disorders, Fourth Edition, Text Revision; FTD: Frontotemporal dementia; GDS: Geriatric depression scale; GLMs: Generalised linear models; MHIS: Modified Hachinski Ischemic Scale; MMSE: Mini-mental state examination; MRI: Magnetic resonance imaging; NINCDS-ADRDA: National Institute of Neurologic and Communicative Disorders and Stroke and the Alzheimer's Disease and Related Disorders Association; NPI: Neuropsychiatric inventory; PD: Parkinson's dementia; SD: Standard deviation

\section{Acknowledgments}

We thanks to all the 81 research centers: The First Affiliated Hospital of Anhui Medical University; Beijing Geriatric Hospital; Beijing Haidian Hospital; Peking Union Medical College Hospital (East); Aerospace Center Hospital; Beijing Chaoyang Hospital, Capital Medical Universit; Beijing Friendship Hospital, Capital Medical University; Fuxing Hospital, Capital Medical University; Xuanwu Hospital, Capital Medical University; China-Japan Friendship Hospital; Peking University First Hospital; The First Affiliated Hospital of Fujian Medical University; Fujian Medical University Union Hospital; Zhongshan Hospital Xiamen University; Provincial Hospital; Foshan Hospital Affiliated to Sun Yatsen University; Guangdong General Hospital; Guangzhou First People's Hospital Guangzhou Medical University; Guangzhou Huiai Hospital; The Third 
Affiliated Hospital of Sun Yat-sen University; Sun Yat-sen Memorial Hospital, Sun Yat-sen University; The First Affiliated Hospital; The Guangxi Zhuang Autonomous Region Jiangbin Hospital; The Affiliated Hospital of Guizhou Medical University; Hainan Province Nongken General Hospital; Hainan Provincial People's Hospital; First Hospital of Qinhuangdao; Hebei General Hospital; No.1 People's Hospital in Shijiazhuang; First Hospital of Hebei Medical University; Kaifeng Central Hospital,; Henan Provincial People's Hospital; The Fifth Affiliated Hospital of Zhengzhou University; Daqing oilfield General Hospital; First affiliated hospital of Harbin medical university; Zhongnan Hospital of Wuhan University; The Third Xiangya Hospital of Central South University; Xiangya Hospital Central South University; The first Bethune Hospital of Jilin University; Northern Jiangsu People's Hospital; The First Affiliated Hospital; People's Hospital of Jiangxi Province; Anshan Changda Hospital; Affiliated Zhongshan Hospital of Dalian University; Dalian Municipal Central Hospital Affillated of Dalian Medical University; The first hospital of China medical university; Shengjing Hospital of China Medical University; Baotou City Central Hospital; Inner Mongolia People's Hospital; Xining City no.1 People's Hospital; Shangdong Provincal Hospital; Qingdao Municipal Hospital; Chinese PLA eighty-eighth hospital; Weihai Municipal Hospital; Shan Xi Provincial People's Hospital; First Hospital of Shanxi Medical University; XiJing Hospital; The First Affiliated Hospital of Xi'an Jiao Tong University; Huashan Hospital, Fudan University; Rui Jin Hospital Shanghai Jiao Tong University School of Medicine; Shanghai Mental Health Center; West China Hospital, Sichuan University; Affiliated Hospital of North Sichuan Medical College; Tianjin An Ding Hospital; Tianjin Huanhu Hospital; Tianjin Medical University General Hospital; Urumqi General Hospital of Lanzhou Military Area Command; Xinjiang Bingtuan Hospital; Traditional Chinese Medicine Hospital of Xinjiang Autonomous Region; The First Affiliated Hospital of Kunming Medical University; The First Affiliated Hospital of Zhejiang University; Sir Run Run Shaw Hospital, School of Medicine, Zhejiang University; Tongde Hospital of Zhejiang Province; Zhejiang Provincial People's Hospital; Kangning Hospital Affiliated to Wenzhou Medical University; The First Affiliated Hospital of Wenzhou Medical University; The Second Affilliated Hospital, Zhejiang University, Medical School; Daping Hospital, Third Military Medical University Chongqing; Southwest Hospital, Third Military Medical University Chongqing; The First Affiliated Hospital of Chongqing Medical University; General Hospital of Ningxia Medical University.

This study was supported by CHINA-CANADA Joint Initiative on Alzheimer's Disease and Related Disorders (81261120571), the Key Project of the National Natural Science Foundation of China (81530036), the National Key Scientific Instrument and Equipment Development Project (31627803), Key medical professional development plan of Beijing Municipal Administration of Hospitals (ZY201301), Mission Program of Beijing Municipal Administration of Hospitals (SML20150801), Beijing Scholars Program and Beijing Municipal Science \& Technology Commission (Z161100000216137).

\section{Funding}

This study is supported by the CHINA-CANADA Joint Initiative on Alzheimer's Disease and Related Disorders (81261120571), Key Medical Professional Development Plan of Beijing Municipal Administration of Hospitals (ZY201301). China National Nature Science Foundation (81530036). Beijing Scholars Program and Beijing Municipal Science \& Technology Commission (Z161100000216137)

\section{Availability of data and materials}

The datasets used and/or analysed during the current study available from the corresponding author on reasonable request.

\section{Authors' contributions}

The work presented here was carried out in collaboration between all authors. JJ and CW conceived and designed the study. SC and FL were major contributors in writing the manuscript. JJ and CW reviewed and edited the manuscript. All authors read and approved the manuscript.

\section{Competing interests}

The authors declare that they have no competing interests.

\section{Consent for publication}

Not applicable.

\section{Ethics approval and consent to participate}

The study protocol was approved by the Ethics Committee of Xuan Wu Hospital Capital Medical University. Written informed consent was obtained from all enrolled participants. Xuan Wu Hospital Capital Medical University has granted ethics approval; the review coding is [2015] 028.

\section{Author details}

'Department of Neurology, Xuan Wu Hospital, Capital Medical University, Beijing, People's Republic of China. ${ }^{2}$ Center of Alzheimer's Disease, Beijing Institute for Brain Disorders, Beijing, People's Republic of China. ${ }^{3}$ Beijing Key Laboratory of Geriatric Cognitive Disorders, Beijing, People's Republic of China. ${ }^{4}$ Neurodegenerative Laboratory of Ministry of Education of the People's Republic of China, Beijing, People's Republic of China.

Received: 2 September 2016 Accepted: 19 January 2017

Published online: 25 January 2017

\section{References}

1. The main data announcement of Sixth national census. 2011. http://www. stats.gov.cn/ztjc/zdtjgz/zgrkpc/dlcrkpc/dcrkpcyw/201104/t20110428_69407. htm, 2011-04-28. Accessed 7 Aug 2015.

2. Jia J, Zhou A, Wei C, Jia X, Wang F, Li F, Wu X, Mok V, Gauthier S, Tang M, et al. The prevalence of mild cognitive impairment and its etiological subtypes in elderly Chinese. Alzheimers Dement. 2014;10(4):439-47. doi:10.1016/j.jalz.2013.09.008.

3. Liu N, Zhang J, Guo L. Alzheimer's disease epidemiological situation. J Liaoning Univ Tradit Chin Med. 2011;01:35-6.

4. Lei T, Ma Y, Nie H, et al. China's current old age dementia prevalence of Meta-analysis. Mod Prev Med. 2012;39(4):809-11. 814.

5. Zhang Y, Xu Y, Nie H, Lei T, Wu Y, Zhang L, Zhang M. Prevalence of dementia and major dementia subtypes in the Chinese populations: a meta-analysis of dementia prevalence surveys, 1980-2010. J Clin Neurosci. 2012;19(10):1333-7. doi:10.1016/j.jocn.2012.01.029.

6. Jia J, Wang F, Wei C, Zhou A, Jia X, Li F, Dong X. The prevalence of dementia in urban and rural areas of China. [Journal Article; Research Support, Non-U.S. Gov't]. Alzheimers Dement. 2014;10(1):1-9. doi:10. 1016/j.jalz.2013.01.012.

7. Costa N, Derumeaux H, Rapp T, Garnault V, Ferlicoq L, Gillette S, Andrieu S, Vellas B, Lamure M, Grand A, et al. Methodological considerations in cost of illness studies on Alzheimer disease. Health Econ Rev. 2012;2(1):18. doi:10. 1186/2191-1991-2-18.

8. Jonsson L, Wimo A. The cost of dementia in Europe: a review of the evidence, and methodological considerations. Pharmacoeconomics. 2009;27(5):391-403.

9. Jones RW, Romeo R, Trigg R, Knapp M, Sato A, King D, Niecko T, Lacey L. Dependence in Alzheimer's disease and service use costs, quality of life, and caregiver burden: the DADE study. Alzheimers Dement. 2015;11(3):280-90. doi:10.1016/j.jalz.2014.03.001

10. Guangzhou Biaodian medical information Co., LTD. Anti-dementia drug market research report. Guangzhou: Industry information service of Minei website, 2012.

11. Gerves C, Chauvin P, Bellanger MM. Evaluation of full costs of care for patients with Alzheimer's disease in France: the predominant role of informal care. Health Policy. 2014;116(1):114-22. doi:10.1016/j.healthpol. 2014.01.001.

12. Tolppanen AM, Taipale H, Purmonen T, Koponen M, Soininen H, Hartikainen S. Hospital admissions, outpatient visits and healthcare costs of communitydwellers with Alzheimer's disease. Alzheimers Dement. 2015;11(8):955-63. doi:10.1016/j.jalz.2014.10.005.

13. Leibson CL, Long KH, Ransom JE, Roberts RO, Hass SL, Duhig AM, Smith CY, Emerson JA, Pankratz VS, Petersen RC. Direct medical costs and source of cost differences across the spectrum of cognitive decline: a populationbased study. Alzheimers Dement. 2015;11(8):917-32. doi:10.1016/j.jalz. 2015.01.007.

14. Keogh-Brown MR, Jensen HT, Arrighi HM, Smith RD. The impact of Alzheimer's disease on the Chinese economy. EBioMedicine. 2016;4:184-90.

15. Reisberg B. Diagnostic criteria in dementia: a comparison of current criteria, research challenges, and implications for DSM-V. J Geriatr Psychiatry Neurol. 2006;19(3):137-46. doi:10.1177/0891988706291083.

16. McKhann G, Drachman D, Folstein M, Katzman R, Price D, Stadlan EM Clinical diagnosis of Alzheimer's disease: report of the NINCDS-ADRDA Work 
Group under the auspices of Department of Health and Human Services Task Force on Alzheimer's Disease. Neurology. 1984;34:939-44.

17. Hachinski VC, Lassen NA, Marshall J. Multi-infarct dementia. A cause of mental deterioration in the elderly. Lancet. 1974;2(7874):207-10.

18. Feng F, Han X, Chen J, et al. The application of activity of daily living scale in dementia screening. J Clin Psychiatry. 2004;04:193-4.

19. Scocco P, Fantoni G, Caon F. Role of depressive and cognitive status in self-reported evaluation of quality of life in older people: comparing proxy and physician perspectives. Age Ageing. 2006;35(2):166-71. doi:10.1093/ ageing/afj026

20. Erkinjuntti T, Bowler JV, DeCarli CS, Fazekas F, Inzitari D, O'Brien JT, Pantoni L, Rockwood K, Scheltens P, Wahlund LO, et al. Imaging of static brain lesions in vascular dementia: implications for clinical trials. Alzheimer Dis Assoc Disord. 1999;13 Suppl 3:S81-90

21. Barber R, Scheltens P, Gholkar A, Ballard C, McKeith I, Ince P, Perry R, O'Brien J. White matter lesions on magnetic resonance imaging in dementia with Lewy bodies, Alzheimer's disease, vascular dementia, and normal aging. J Neurol Neurosurg Psychiatry. 1999;67(1):66-72.

22. Folstein MF, Folstein SE, McHugh PR. "Mini-mental state". A practical method for grading the cognitive state of patients for the clinician. J Psychiatr Res. 1975:12(3):189-98.

23. McKhann GM, Albert MS, Grossman M, Miller B, Dickson D, Trojanowski JQ. Clinical and pathological diagnosis of frontotemporal dementia: report of the Work Group on Frontotemporal Dementia and Pick's Disease. Arch Neurol. 2001;58(11):1803-9.

24. McKeith IG, Dickson DW, Lowe J, Emre M, O'Brien JT, Feldman H, Cummings J, Duda JE, Lippa C, Perry EK, et al. Diagnosis and management of dementia with Lewy bodies: third report of the DLB Consortium. Neurology. 2005; 65(12):1863-72. doi:10.1212/01.wnl.0000187889.17253.b1.

25. Emre M, Aarsland D, Brown R, Burn DJ, Duyckaerts C, Mizuno Y, Broe GA Cummings J, Dickson DW, Gauthier S, et al. Clinical diagnostic criteria for dementia associated with Parkinson's disease. Mov Disord. 2007:22(12):1689. doi:10.1002/mds.21507. -1707, 1837.

26. Wang G, Cheng Q, Zhang S, Bai L, Zeng J, Cui PJ, Zhang T, Sun ZK, Ren RJ Deng $Y L$, et al. Economic impact of dementia in developing countries: an evaluation of Alzheimer-type dementia in Shanghai. China Alzheimers Dis. 2008;15(1):109-15.

27. van den Berg B, Bleichrodt $H$, Eeckhoudt $L$. The economic value of informal care: a study of informal caregivers' and patients' willingness to pay and willingness to accept for informal care. Health Econ. 2005;14(4):363-76. doi:10.1002/hec.980

28. Hurd MD, Martorell P, Delavande A, et al. Monetary costs of dementia in the United States. N Engl J Med. 2013;368(14):1326-34.

29. Cummings JL. The Neuropsychiatric Inventory: assessing psychopathology in dementia patients. Neurology. 1997;48(5 Suppl 6):S10-16.

30. Mesterton J, Wimo A, By A, Langworth S, Winblad B, Jönsson L. Cross sectional observational study on the societal costs of Alzheimer's disease. Curr Alzheimer Res. 2010;7(4):358-67.

31. Alzheimer's Disease International. World Alzheimer Report 2010: The global economic impact of dementia. Available at http://www.alz.co.uk/research/ files/WorldAlzheimerReport2010.pdf. Accessed 21 Apr 2015.

32. Wucherer D, Eichler T, Kilimann I, Hertel J, Michalowsky B, Thyrian JR, Teipel S, Hoffmann W. Antidementia drug treatment in people screened positive for dementia in primary care. J Alzheimers Dis. 2015;44(3):1015-21. doi:10. 3233/JAD-142064

33. Chan KY, Wang W, Wu JJ, Liu L, Theodoratou E, Car J, Middleton L, Russ TC, Deary IJ, Campbell H, et al. Epidemiology of Alzheimer's disease and other forms of dementia in China, 1990-2010: a systematic review and analysis. Lancet. 2013;381(9882):2016-23. doi:10.1016/S0140-6736(13)60221-4.

34. Dong MJ, Peng B, Lin XT, Zhao J, Zhou YR, Wang RH. The prevalence of dementia in the People's Republic of China: a systematic analysis of 19802004 studies. Age Ageing. 2007;36(6):619-24. doi:10.1093/ageing/afm128.

35. Mihaylova B, Briggs A, O'Hagan A, Thompson SG. Review of statistical methods for analysing healthcare resources and costs. Health Econ. 2011; 20(8):897-916. doi:10.1002/hec.1653.

36. Luo HQ, Qian JH, Wu K, Cao PY, Ren XH. Regional Differences and Determinants of Self-rated Health in Elderly. Sichuan Da Xue Xue Bao Yi Xue Ban. 2016:47(2):248-52.
37. Shen Y, Yeatts DE, Cai T, Yang PQ, Cready CM. Social capital and self-rated health among middle-aged and older adults in China: a multilevel analysis. Res Aging. 2014;36(4):497-521. doi:10.1177/0164027513505624.

38. Song Y, Wang J. Overview of Chinese research on senile dementia in mainland China. Ageing Res Rev. 2010;9 Suppl 1:S6-12. doi:10.1016/j.arr.2010.08.007.

39. Diagnosis and treatment of dementia in neurology outpatient departments of general hospitals in China. Alzheimers Dement. 2016;12(4):446-453.

\section{Submit your next manuscript to BioMed Central and we will help you at every step:}

- We accept pre-submission inquiries

- Our selector tool helps you to find the most relevant journal

- We provide round the clock customer support

- Convenient online submission

- Thorough peer review

- Inclusion in PubMed and all major indexing services

- Maximum visibility for your research

Submit your manuscript at www.biomedcentral.com/submit
Biomed Central 